\title{
Análise da acessibilidade dos portadores de deficiência física nas escolas da rede pública de Passo Fundo e o papel do fisioterapeuta no ambiente escolar
}

\author{
Analysis of the accessibility to the public schools in Passo Fundo by the \\ disabledchildren and the Physical Therapist role at school surrounding
}

Carina Tagliari', Francesca Três², Sheila Gemelli de Oliveira ${ }^{3}$

\begin{abstract}
RESUMO
Objetivo: Este estudo objetivou analisar as escolas da rede pública de Passo Fundo/RS quanto à acessibilidade e orientar os funcionários, alunos Portadores de Deficiência Física (PDF) e seus familiares de como estes alunos devem se portar no ambiente escolar. Método: Utilizamos um questionário com objetivo de coletar dados sobre condições de acesso e permanência dos PDF nas dependências das escolas. Visitamos 63 escolas incluídas nesta pesquisa, explicamos os objetivos do estudo aos responsáveis de cada escola, aplicamos um questionário e oferecemos um folder que consta de procedimentos e estratégias de inclusão dos PDF e de como estes devem se portar no ambiente escolar. Resultados: Ao analisarmos os dados, encontramos 22 alunos PDF em 14 escolas. A maioria das escolas analisadas não tem acessibilidade necessária para estes alunos, não existem projetos para a eliminação de barreiras arquitetônicas e ambientais, não há presença de profissional capacitado para trabalhar com os funcionários quanto aos procedimentos e estratégias de inclusão dos alunos PDF e para orientá-los de como se portar no ambiente escolar. Conclusão: Nosso estudo mostra a total falta de estrutura, informação e treino dos funcionários e dos pais em relação às necessidades dos alunos PDF que estão sob sua responsabilidade. Sugerimos que palestras explicativas e demonstrativas sobre esse assunto pode melhorar a qualidade de vida desses alunos.
\end{abstract}

Unitermos: Crianças portadoras de deficiência, Estruturas de acesso, Fisioterapia.

Citação: Tagliari C, Três F, Oliveira SG. Análise da acessibilidade dos portadores de deficiência física nas escolas da rede pública de Passo Fundo e o papel do fisioterapeuta no ambiente escolar. Rev Neurocienc 2006; 14(1):010-014.

\section{SUMMARY}

Objective: We had as objective to analyze the schools as accessibility and to orient the school staff, PDF students and the relatives of disabled children how these students must act at school surroundings. Method: This research used a questionnaire that had as goals to get information about disabled children permanence and access conditions at school surroundings. Results: Visiting 63 schools included in this research, we explained the objectives from this study to the responsible from each school, soon we applied the questionnaires and offered an explanatory folder that consists of procedures and inclusion strategies of the disabled children and how these people must act at school surroundings. We analyzed the information and we found 22 disabled students in 14 schools. The study showed that the majority of the schools analyzed do not have the necessary accessibility for these students, there are no projects to eliminate the architectural and environmental barriers, and there is nobody qualified to provide inclusion strategies and procedures for disabled students and school staff, or even to guide them how to behave in the school surroundings.

Trabalho realizado: Faculdade de Fisioterapia da Universidade de Passo Fundo - RS

1 - Acadêmica de Fisioterapia da Universidade de Passo Fundo.

2 - Acadêmica de Fisioterapia da Universidade de Passo Fundo,

3 - Docente do Curso de Fisioterapia da Universidade de Passo Fundo, Mestre em Gerontologia Biomédica.

Endereço para Correspondência: Francesca Três - Av. Sarandi, 699, Centro - Rondinha - RS, CEP:99.590.000 - E-mail: ecatres@yahoo.com.br 
Conclusions: Our study disclosed a complete lack of structure, information, and training of school staff and parents regarding the needs of disabled children that they have under their responsibility. We suggest that some explanatory and demonstrative lectures on this issue could improve the quality of life of this students.

\section{Keywords. Disabled children, Architectural accessibility, Physical therapy.}

Citation: Tagliari C, Três F, Oliveira SG. Analysis of the accessibility to the public schools in Passo Fundo by the disabled persons and the physiotherapist role at scholar surrounding. Rev Neurocienc 2006; 14(1):010-014.

\section{INTRODUÇÃO}

A oportunidade que o ser humano tem de crescer leva a sua valorização onde ele pode se manifestar, expandir e desenvolver suas atividades, mas esta busca se torna limitada pelos obstáculos arquitetônicos junto às instituições públicas de ensino, onde o deficiente, muitas vezes, não tem condições de acesso e/ ou permanência na mesma ${ }^{1}$.

Deficiência física é todo comprometimento da mobilidade, coordenação motora geral ou da fala, causado por lesões neurológicas, neuromusculares e ortopédicas ou ainda por má formação congênita ou adquirida ?

O último censo realizado pelo IBGE ${ }^{3}$, revelou que o Brasil tem 26,5 milhões de pessoas portadoras de necessidades especiais. Desse total, cerca de $14 \%$ são exclusivamente deficientes físicos.

No Rio Grande do Sul, numa população de quase 10 milhões de habitantes, 15,01 \% deles, ou seja, aproximadamente 1.501 .000 pessoa, possuem algum tipo de deficiência (IBGE 2000) e constituindo-se, portanto, alvo de políticas públicas inclusivas ${ }^{4}$.

De acordo com IBGE de 2000 a população estimada em Passo Fundo no ano de 2004 foi de 182.233, com número total de pessoas com algum tipo de deficiência de 23.852 (13,0887\%). A DAPNE - PF atende 1.753 portadores de necessidades especiais e outras instituições atendem 1.060 , totalizando 2.813 cadastrados ${ }^{5}$.

Conforme Censo Escolar de 2004, no Rio Grande do Sul há 37.734 alunos com deficiência, destes, 12.193 estão incluídos em escolas públicas regulares sendo que 8.301 possuem apoio pedagógico ${ }^{6}$.

A Lei $n^{\circ}$ 10.098, de 19 de dezembro de 2000 estabelece normas gerais e critérios básicos para a promoção da acessibilidade das pessoas portadoras de deficiência ou com mobilidade reduzida, mediante a supressão de barreiras e de obstáculos nas vias e espaços públicos, no mobiliário urbano, na construção e reforma de edifícios e nos meios de transportes e de comunicação ?

Nesta mesma lei consta que para possibilitar o acesso de pessoa com deficiência física, todas as escolas devem eliminar suas barreiras arquitetônicas tendo ou não alunos com deficiência matriculado no momento ?

Assim como mostra a norma de acessibilidade atualizada para PDF nas escolas, no item "8.6.2 Deve existir pelo menos uma rota acessível interligando o acesso de alunos às áreas administrativas, de prática esportiva, de recreação, de alimentação, salas de aula, laboratórios, bibliotecas, centros de leitura e demais ambientes pedagógicos. Todos estes ambientes devem ser acessíveis" 7 .

Para Neto ${ }^{8}$ existem três tipos de barreiras: Físicas: degraus; Sistêmicas: estabelecimentos de ensino que não oferecem serviços assistivos - tais como anotação de aulas para alunos que não conseguem fazê-lo eles mesmos; atitudinais: em forma de preconceitos.

Lorenzini ${ }^{9}$ diz que a pessoa portadora de deficiência física passa longos períodos na posição sentada e devido a seus padrões posturais inadequados é muitas vezes prejudicada no seu rendimento geral devido à falta de mobiliário adequado que lhe proporcione oportunidades para desenvolver o máximo de seu potencial.

Objetivando-se uma educação inclusiva em todos os aspectos, as tendências atuais apresentam a busca de uma integração e/ou inter-relação entre os profissionais das áreas da educação e da saúde. A Lei de Diretrizes e Bases da Educação de 1996, vai ao encontro destas tendências quando prevê a inserção de serviços de apoio especializado na escola regular, atendendo as peculiaridades da clientela de educação especial ${ }^{8}$.

Segundo Carvalho ${ }^{10}$, a Conferência Mundial sobre Educação para Todos realizada em Jomtien/Tailândia 1990, ao abordar a importância de universalizar o acesso à educação e promover a eqüidade, destacou que as necessidades básicas de aprendizagem das pessoas portadoras de necessidades especiais requerem atenção especial e é preciso tomar medidas que garantam a igualdade de acesso à educação aos portadores de todo e qualquer tipo de necessidade especial, como parte integrante do sistema educativo.

De acordo com Estatuto da Criança e do Adolescente a escola não pode recusar matrícula de criança com deficiência física ou mental, deve matricular e procurar se adaptar e capacitar seus professores o quanto antes ${ }^{11}$

Ribeiro ${ }^{12}$, diz que quando os recursos existentes na própria escola mostrarem-se insuficientes para melhor compreender as necessidades educacionais dos alunos e identificar os apoios indispensáveis, a escola poderá recorrer a uma equipe multiprofissional.

Lorenzini ${ }^{9}$ menciona que cabe ao fisioterapeuta instruir o professor sobre o posicionamento adequado 
para determinada deficiência física; assim como orientá-lo na seleção e uso de equipamentos, mobiliários, dispositivos de suporte, adaptações e facilitação dos padrões posturais, bem como condições de funcionalidade do aluno, tanto no ambiente em sala de aula como em atividades extra-classe, como passeios, jogos recreacionais, enfim, em qualquer atividade.

O Fisioterapeuta tem papel preponderante no ambiente escolar uma vez que poderá propor mudanças e inovações não somente externa como internamente, possibilitando melhores condições de acesso e permanência do portador de deficiência física proporcionando sua inclusão no ambiente escolar e assim melhorando a sua qualidade de vida ${ }^{1}$.

\section{MÉTODO}

A estratégia metodológica utilizada nesta pesquisa é o estudo transversal, uma vez que em determinado momento foram coletadas informações referentes a uma amostra de indivíduos de uma população ${ }^{13}$, para a análise da acessibilidade dos portadores de deficiência física nas escolas da rede pública da zona urbana de Passo Fundo/RS.

Utilizamos um questionário aplicado, o qual foi respondido pela diretora ou responsável pela escola. Este questionário teve como objetivo coletar dados sobre as condições de acesso e permanência dos PDF nas dependências das escolas e se os mesmos recebem orientações de como se portar no ambiente escolar.

Entramos em contato com a $7^{\mathrm{a}}$ Coordenadoria Estadual de Educação e com a Secretaria Municipal de Educação, no setor pedagógico, onde apresentou-se o projeto da pesquisa e solicitou-se a autorização para a sua realização e listagem das escolas estaduais e municipais da zona urbana de Passo Fundo.

Ao visitar as 35 escolas municipais e as 28 escolas estaduais incluídas nesta pesquisa, explicamos os objetivos do presente estudo aos responsáveis de cada escola, em seguida aplicamos o questionário aos mesmos. Após o questionamento oferecemos um folder explicativo que consta de procedimentos e estratégias de inclusão dos PDF e de como estes devem se portar no ambiente escolar. Ao término das entrevistas, analisamos os dados e retornamos nas escolas com alunos PDF e realizamos palestra explicativa e demonstrativa sobre posturas e cuidados que estes alunos devem ter para uma melhor qualidade de vida.

\section{RESULTADOS E DISCUSSÃO}

Das 35 escolas municipais 6 tem alunos PDF, 2 são cadeirantes e 4 com mobilidade reduzida; 13 escolas tem prédio com mais de um piso; em 16 escolas o aluno cadeirante não consegue chegar até a entrada da mesma; há rampa de acesso em apenas 9; em 11 tem acesso principal, da calçada para dentro da escola (Figura 1).

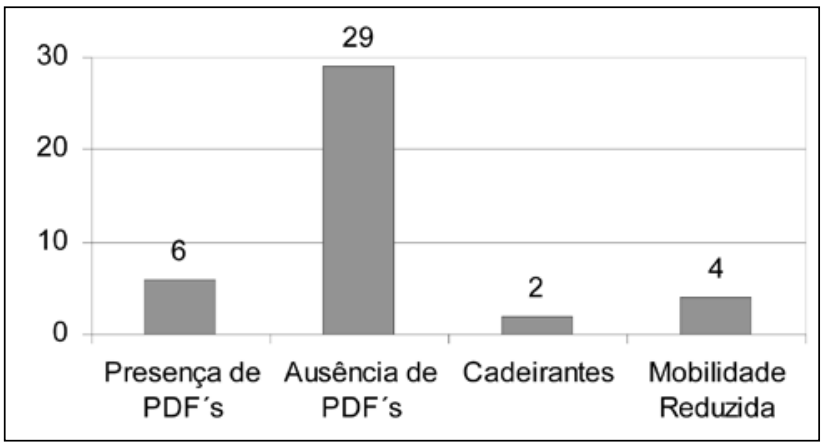

Figura 1. Distribuição quanto à presença de alunos Portadores de Deficiência Física (PDF) nas Escolas Municipais de Passo Fundo - RS.

De acordo com Machado ${ }^{14}$, as crianças portadoras de deficiência física têm capacidade cognitiva semeIhante a de colegas e podem realizar as atividades com bom desempenho desde que as limitações físicas sejam contornadas. A grande limitação é o deslocamento na escola, na maioria dos casos há dificuldades em relação às edificações da escola e dificuldades em relação às escadas.

Quanto aos acessos secundários (Figura 2), 18 escolas tem acesso para as salas de aula, 9 para os sanitários, 15 para as lancherias e 14 para as áreas de recreação.

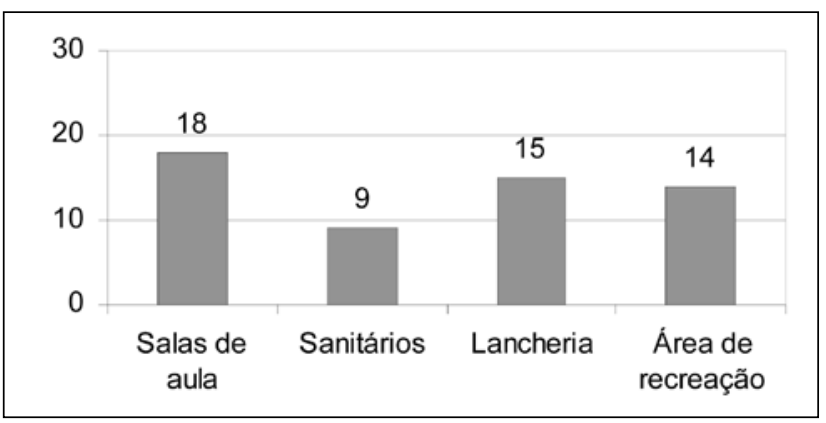

Figura 2. Presença de acessos secundários nas Escolas Municipais de Passo Fundo - RS.

A Lei n $7.853 / 89$ transfere para Estados e Municípios a responsabilidade pela adoção de normas que eliminem as barreiras de acesso das pessoas portadoras de deficiência a edificações, espaços urbanos e meios de transporte ${ }^{15}$.

Conforme a norma de acessibilidade atualizada para PDF nas escolas, no item "8.6.4 Pelo menos 5\% dos sanitários, com no mínimo um sanitário para cada sexo, de uso dos alunos, devem ser acessíveis, conforme seção 7. Recomenda-se, além disso, que pelo menos outros $10 \%$ sejam adaptáveis para acessibilidade.", no item "8.6.7 Nas salas de aula, quando houver mesas individuais para alunos, pelo menos $1 \%$ do total de 
mesas, com no mínimo uma para cada duas salas de aula, deve ser acessível a P.C.R. ..." e no item "8.6.9 Todos os elementos do mobiliário urbano da edificação como bebedouros, guichês e balcões de atendimento, bancos de alvenaria, entre outros, devem ser acessíveis, conforme seção 9"7.

Quanto a estrutura dos banheiros, 3 escolas tem vaso adaptado, 5 escolas tem barras ou suportes firmes próximos ao vaso, em 31 escolas a estrutura das pias permite aos PDF terem acesso a torneira.

Em apenas 1 escola há classe adaptada, mas nesta escola os alunos cadeirantes não tem acesso a sala de aula pela presença de uma escadaria. Esta escola tem lugar para um elevador, o qual nunca foi colocado.

Para Lorenzini ${ }^{9} \mathrm{o}$ primeiro requisito para o bom mobiliário é ajustar a cadeira às dimensões anatômicas do usuário. Com adequação do mobiliário cadeira-mesa estaremos prevenindo as retrações de tecidos moles, as deformidades esqueléticas, estaremos proporcionando condições para uma melhor ventilação pulmonar, e oferecendo condições de melhora de seu tônus postural e de melhora ou manutenção de déficit muscular.

Em 24 escolas a largura das portas possibilita a entrada e saída dos alunos com cadeira de rodas; a altura das maçanetas são acessíveis em 34 escolas; os interruptores de luz são acessíveis em 30 escolas; e em apenas 4 os alunos cadeirantes conseguem usar o bebedouro.

Nas escolas que tem alunos PDF, 4 deles participam das atividades físicas ou de recreação.

A educação física contribui de forma marcante para a discriminação do deficiente físico na escola. Se o aluno for excluído da prática da atividade física regular possivelmente isso será um estímulo para a exclusão deste da escola, podendo acarretar, graves problemas para o seu desenvolvimento ${ }^{1}$.

Em apenas 4 escolas existe algum projeto para eliminação de barreiras arquitetônicas e ambientais. Das 6 escolas com alunos PDF, 4 realizam trabalhos com os funcionários quanto aos procedimentos e estratégias de inclusão destes alunos. Dos 6 alunos PDF, apenas 3 recebem orientações de como se portar no ambiente escolar, e estas orientações são dadas somente pelo corpo docente, e em nenhuma escola há presença de fisioterapeuta.

O fisioterapeuta pode desenvolver programas de treinamento para instruir o educador que lida com classes de estudantes com deficiências físicas, tais como técnicas de posicionamento ou métodos para auxiliar na independência funcional da criança ${ }^{1}$.

Das 28 escolas estaduais 8 tem alunos PDF. Foram encontrados 16 alunos PDF, 8 cadeirantes e $8 \mathrm{com}$ mobilidade reduzida (figura 3 ).

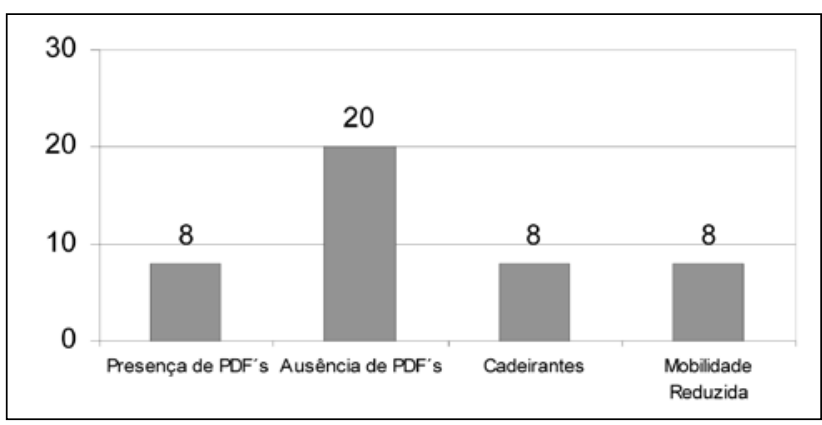

Figura 3. Distribuição quanto à presença de alunos Portadores de Deficiência Física (PDF) nas Escolas Estaduais de Passo Fundo-RS.

A Constituição Federal mostra no seu Art. 208, inciso III - Atendimento educacional às pessoas portadoras de deficiência, preferencialmente na rede regular de ensino ${ }^{4}$.

Em 17 escolas tem prédio com mais de um piso; em 6 escolas o aluno cadeirante não consegue chegar até a entrada da mesma; há rampa de acesso em apenas 16; em 21 tem acesso principal (da calçada para dentro da escola).

De acordo com Machado ${ }^{14}$ as maiores dificuldades encontradas dentro da escola são o deslocamento e o subir escadas.

Quanto aos acessos secundários, 15 escolas tem acesso para as salas de aula, 12 para os sanitários, 11 para as lancherias e 17 para as áreas de recreação (Figura 4).

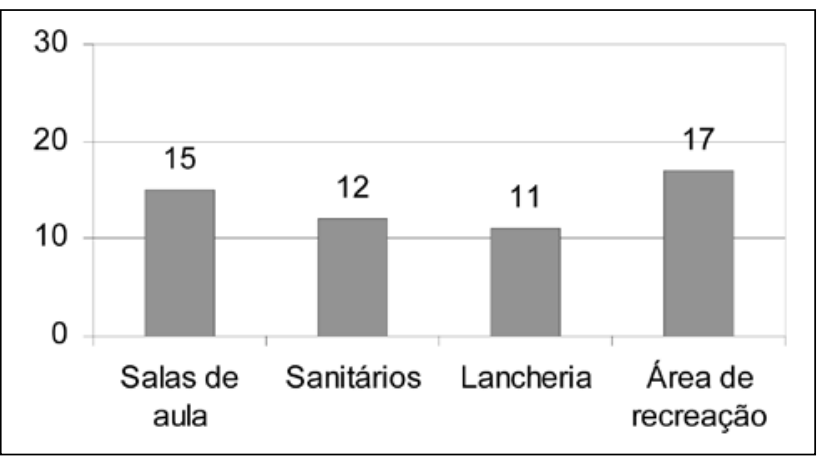

Figura 4. Presença de acessos secundários nas Escolas Estaduais de Passo Fundo - RS.

Quanto a estrutura dos banheiros, 1 escolas tem vaso adaptado, 3 escolas tem barras ou suportes firmes próximos ao vaso, em 24 escolas a estrutura das pias permite aos PDF terem acesso a torneira. Em apenas 1 escola há classe adaptada.

Para Quevedo ${ }^{16}$ o que impede a continuidade à formação cultural dos PDF é a impossibilidade de acesso ao interior das instituições, a circulação no ambiente interno das instituições, a inadequabilidade dos sanitários, das salas de aula e mobiliário. 
Em 26 escolas a largura das portas possibilita a entrada e saída dos alunos com cadeira de rodas; a altura das maçanetas são acessíveis em 27 escolas; os interruptores de luz são acessíveis em 27 escolas; e em apenas 9 os alunos cadeirantes conseguem usar o bebedouro.

Nas escolas que tem alunos PDF, 11 deles participam das atividades físicas ou de recreação.

Conforme Machado ${ }^{14}$, poderia o fisioterapeuta atuar orientando sobre as condições motoras do indivíduo ao educador físico e demais professores para que situações como essas deixem de ocorrer, efetivando o trabaIho de inclusão do aluno portador de deficiência física em todas as atividades realizadas na escola e, assim, torná-lo mais participativo no grupo de colegas.

Em apenas 1 escola existe projeto para eliminação de barreiras arquitetônicas e ambientais. Das 8 escolas com alunos PDF, 4 realizam trabalhos com os funcionários quanto aos procedimentos e estratégias de inclusão destes alunos. Dos 16 alunos PDF, apenas 8 recebem orientações de como se portar no ambiente escolar, e estas orientações são dadas somente pelo corpo docente, e em nenhuma escola há presença de fisioterapeuta.

Segundo Lorenzini ${ }^{9}$ o papel do fisioterapeuta, no que se refere ao trabalho com crianças portadoras de deficiência física em escolas regulares, visa transmitir habilidades fundamentais para contribuir com o profes- sor e outros profissionais, tentando minimizar as dificuldades dessas crianças objetivando um desenvolvimento máximo de suas potencialidades.

\section{CONCLUSÃO}

Ao final do presente trabalho, após realizadas as entrevistas nas escolas da rede pública de Passo Fundo, observamos que a maioria das instituições de ensino não estão adaptadas aos deficientes físicos e apenas uma escola foi construída com projeto de favorecer a acessibilidade dos PDF.

Com este trabalho verificamos que a maioria das escolas não tem projetos para eliminação das barreiras arquitetônicas e ambientais das mesmas, o que, provavelmente, não ocorrerá nos próximos 5 anos.

A presente pesquisa constatou que poucos alunos portadores de deficiência física freqüentam as escolas públicas, talvez por não fornecerem condições mínimas de acessibilidade.

Algumas dificuldades encontradas foram quanto à falta de preparo do corpo docente para trabalhar e orientar os alunos PDF, tanto nas atividades físicas ou de recreação, como nas atividades em sala de aula, com relação a postura, pois não existe nenhum treinamento que os habilite para desempenhar estas funções. Sendo que nas mesmas não há presença de fisioterapeutas.

\section{REFERÊNCIAS BIBLIOGRÁFICAS}

1. Costa J. Principais obstáculos para integração dos Portadores de Necessidades nas escolas da rede pública estadual de Passo Fundo (Monografia) Santa Maria: Universidade Federal de Santa Maria, 2004, 54p.

2. Associação do Deficiente Físico Vale do Rio Pardo. Manual para Inclusão Social das Pessoas com Deficiência: Um mundo para todos. Vale do Rio Pardo: ADEFI.VRP, 2004

3. Dados Estatísticos. IBGE. (citado em 04/2005). Disponível em: http://www.ibge.com.br.

4. Fundação de Articulação e Desenvolvimento de Políticas Públicas para PPD'S E PPAH'S no RS. Guia de inclusão social: responsabilidade de todos. Porto Alegre: Governo do Estado do Rio Grande do Sul, 2005.

5. Divisão de Atenção aos Portadores de Necessidades Especiais. Portadores de Deficiências. Passo Fundo: Cadastros da DAPNE, 2005.

6. Cavalheiro R. Escolas despreparadas para o aluno especial. Porto Alegre Zero Hora, 22 abr. 2005

7. Norma Brasileira 9050:2004, Item Escolas, p.87. ABNT. (última atualização05/2004; citado em 03/2006). Disponível em: www.mj.gov.br/sedh/ct/ CORDE/dpdh/corde/ABNT/NBR9050-31052004.pdf

8. Neto AR. Direitos do Portador de Necessidades Especiais. São Paulo: Fiúza, 2002, 361p
9. Lorenzini MV. O papel do Fisioterapeuta em Classe Especial de Crianças Portadoras de Deficiência Física. Fisioter Mov 1992; 4: 17-25.

10. Carvalho RE. Uma Promessa de Futuro: Aprendizagem para Todos e por Toda a Vida. Porto Alegre: Mediação, 2002

11. Sala de aula: a escola que é de todas as crianças. 182 ed. Revista Escola. (citado em 05/2005). Disponível em: < http://www.revistaescola.abril.com. $\mathrm{br} /$ portaldeacesso.com/links/html>

12. Ribeiro MLS. Perspectivas da Escola Inclusiva: Algumas Reflexões. In Baumel RCC, Ribeiro MLS. Educação Especial: Do Querer ao Fazer. São Paulo: Avercamp, 2003

13. Bicalho GG, Barros Filho AA. Iniciação Cientifica: como elaborar um projeto de pesquisa. Rev Ciênc Med 2003; 12: 325-373.

14. Machado F. As dificuldades do aluno portador de deficiência física no processo de adaptação social em escola regular no município de Porto Alegre. (Monografia). Canoas: Universidade Luterana do Brasil, 1999, 79p.

15. Coordenadoria Nacional para Integração de Pessoa Portadora de Deficiência. Município \& Acessibilidade. Rio de Janeiro: CORDE, 1998

16. Quevedo AEP. Obstáculos à qualidade de vida do deficiente físico: estudo de caso junto às instituições públicas de ensino. (Dissertação). Santa Maria: Universidade Federal de Santa Maria, 2003, 118p. 